\title{
Incidence and risk factors for thrombocytopenia in the intensive care units of a tertiary hospital in northern India
}

\author{
C Mehta, ${ }^{1}$ MBBS, DNB, FNB; J V George, ${ }^{1}$ RN/RM; Y Mehta, ${ }^{1}$ MBBS, MD, MNAMS, FRCA; M T Ali, ${ }^{1}$ MBBS, MD, EDIC; \\ M K Singh, ${ }^{2}$ MSc (Biostatistics)
}

${ }^{1}$ Institute of Critical Care and Anesthesia, Medanta - The Medicity, Gurgaon, Haryana, India

${ }^{2}$ Institute of Clinical Research, Medanta - The Medicity, Gurgaon, Haryana, India

Corresponding author: J V George (jobygeorge05@hotmail.com)

\begin{abstract}
Background. In Western countries, incidence of thrombocytopenia in intensive care units (ICUs) has been found to be 13 - $44 \%$. We chose to study the incidence, risk factors and transfusion requirements of thrombocytopenia in tertiary care ICUs in northern India.

Objective. To study the incidence and risk factors of thrombocytopenia in a mixed ICU.

Methods. This prospective observational 6-month cohort study was conducted in two 22-bedded medical-surgical ICUs. Patients aged 18 years or older with an ICU stay of at least 2 days were included.

Results. Thrombocytopenia (<150 000/dL) occurred in $190(38 \%)$ of the 500 patients studied. Thrombocytopenia was present on admission in 41 (8\%) patients. Of the remaining patients, 149 (32\%) developed new-onset thrombocytopenia (NOT) - thrombocytopenia developing in patients with platelet count more than $150000 / \mathrm{U}$ on admission - during ICU stay. Incidence and prevalence were $30 \%$ and $38 \%$, respectively. ICU mortality was $13 \%$. Thrombocytopenia was commonly associated with sepsis, disseminated intravascular coagulation, heparin and certain antibiotics. Cause could not be established in 10 patients. Underlying coronary artery disease and sepsis correlated with thrombocytopenia. Mortality was higher in patients with NOT $(15.4$ v. $8.7 \%, p=0.003)$. Compared with non-thrombocytopenic patients, patients with NOT required more blood product transfusions $(57.7$ v. $38.4 \%, p=0.000)$ and mechanical ventilation $(23.5$ v. $13.5 \%, p=0.008)$. No difference was observed in length of hospital stay and bleeding risk between the two groups.

Conclusion. We found incidence and prevalence of thrombocytopenia in the ICU comparable with internationally reported figures. NOT was associated with higher mortality and morbidity and may be considered as a marker of disease severity.
\end{abstract}

S Afr J Crit Care 2016;32(1):28-31. DOI:10.7196/SAJCC.2016.v32i1.234

Thrombocytopenia is one of the most commonly observed laboratory abnormalities in the intensive care unit (ICU) ${ }^{[1]}$ with incidence ranging from $13 \%$ to $44 \%$. ${ }^{[1]}$ Thrombocytopenia has been found to predispose patients to increased bleeding risk, increasing morbidity and mortality. ${ }^{[2]}$ It is also considered a marker of disease severity. ${ }^{[3]}$

The aetiology of thrombocytopenia in the ICU is multifactorial. Sepsis, malignancy, presence of invasive catheters and various medications such as heparin and antibiotics have been found associated with thrombocytopenia. Because of the many confounding factors, establishing the cause of thrombocytopenia in critically ill patients is challenging. ${ }^{[4,5]}$

Different aspects of thrombocytopenia have been examined in various studies. The majority of these studies are Western, retrospective or have a small sample size. Until now, Indian literature has been silent on the prevalence of thrombocytopenia in the ICU. A Korean group recently evaluated thrombocytopenia in the $\mathrm{ICU}^{[6]}$ the sole study from Asian countries so far. This prompted us to study the incidence, risk factors and impact on outcome of thrombocytopenia in the mixed ICU of our tertiary, multispecialty hospital in northern India.

\section{Methods}

This was a prospective, observational cohort study. Consecutive patients aged 18 years or older admitted to our medical-surgical ICU for at least 2 days were included. The study was conducted over a period of 6 months (October 2012 - March 2013). Patients were excluded if they had history of cardiac surgery, pregnancy, platelet disorders, haematological malignancy, recent chemotherapy, splenectomy, ICU stay of $<48$ hours, were $<18$ years of age or had a history of readmission to the ICU during the same hospital stay. Platelet counts were measured daily from ICU admission to ICU discharge.

The necessary approvals were obtained from the Medanta Institutional Review Board (Ref. no.: 198/2012). Informed consent was obtained from the patients or their relatives prior to data collection. The study was registered at the Clinical Trial Registry of India (CTRI) (Ref. no.: CTRI/2012/05/002620).

The primary study objective was to assess thrombocytopenia incidence and associated risk factors. Secondary objectives were to study the effect of thrombocytopenia on bleeding risk and transfusion requirements in the ICU. The outcomes of length of hospital stay, ICU discharge and mortality were also studied.

\section{Definitions}

Thrombocytopenia was defined as two or more consecutive platelet counts $<150000 / \mu \mathrm{L}$, obtained at least 12 hours apart. Lowest platelet count during the ICU stay was considered the nadir platelet count. Disseminated intravascular coagulation (DIC) was defined by presence of elevated D-dimer along with two of the following criteria: prolonged prothrombin time, increased fibrinogen degradation products, and decreased fibrinogen or platelet counts. Drug-induced thrombocytopenia was considered present if there was resolution of thrombocytopenia after discontinuation of the suspected drug.

Heparin-induced thrombocytopenia (HIT) was diagnosed using $4 \mathrm{~T}$ scoring (Table 1) and if there was recovery in platelet count after discontinuation of unfractionated heparin or low-molecular-weight heparin. Major bleeding was defined as any intracranial bleed or any bleed associated with a fall in haemoglobin by at least $2 \mathrm{~g} / \mathrm{dL}$. 


\section{Data collection}

The following variables were recorded: general demographic characteristics including age, gender, comorbidities, reason for ICU admission; severity of illness by APACHE (Acute Physiology and Chronic Health Evaluation) IIscorecalculation; biochemical parameters of completeblood count, liver function tests, kidney function tests, coagulation assays and any other special blood investigations if conducted; a comprehensive list of drugs patients were receiving in ICU; various interventions performed in ICU such as mechanical ventilation and haemodialysis; frequency and products of blood transfusions, if any; complications such as bleeding episodes or thromboembolic events; and any use of vasopressors.

\section{Statistical analysis}

Analysis included profiling of patients for different demographic and clinical parameters, comorbidity and risk factors. Descriptive analysis of quantitative data was expressed as means and standard deviations (SDs). Ordinal data were expressed as percentages, medians and ranges. Data normality was tested using the Kolmogorov-Smirnov test. For quantitative parameters satisfying normality assumptions, the independent Student's $t$-test was used to draw inferences between groups. Cross-tables were generated to illustrate the relationships between parameters and comparisons were made using the $\chi^{2}$ test. Stepwise multiple logistic regressions were performed for parameters. A $p$-value of $<0.05$ was considered statistically significant. The entire statistical analysis was performed using SPSS software version 19.0 (IBM Corp., USA).

\section{Results}

A total of 500 patients were enrolled in the study. Of these, 41 (8.2\%) had thrombocytopenia on admission. Of the remaining 459 patients, 149 (32.5\%) had new-onset thrombocytopenia (NOT) during their ICU stay. The median APACHE II score at the time of ICU admission was 11.0 (interquartile range (IQR) $1-32$ ), and the predicted death rate was $5-10 \%$. Median length of ICU stay was 4 days (IQR 1 - 35). Median length of hospital stay was 11 days (IQR 1 - 149). Thrombocytopenia incidence was $30 \%$ and prevalence during the study period was $38 \%$. ICU mortality was $13 \%$. The baseline patient demographic profile is shown in Table 2.

\section{Table 1. The 4T scoring system of HIT ${ }^{[7]}$}

\begin{tabular}{|c|c|c|c|}
\hline & 2 & 1 & $\mathbf{0}$ \\
\hline Thrombocytopenia & $>50 \%$ platelet fall to nadir $\geq 20$ & $30-50 \%$ platelet fall or nadir $10-19$ & $<30 \%$ platelet fall or nadir $<10$ \\
\hline $\begin{array}{l}\text { Timing* of onset of platelet fall } \\
\text { (or other sequelae of HIT) }\end{array}$ & $\begin{array}{l}\text { Days } 5-10 \text {, or } \leq \text { day } 1 \text { with recent } \\
\text { heparin (past } 30 \text { days) }\end{array}$ & $\begin{array}{l}>\text { day } 10 \text { or timing unclear; or < day } 1 \text { with } \\
\text { recent heparin (past } 31-100 \text { days) }\end{array}$ & $<$ day 4 (no recent heparin) \\
\hline Thrombosis or other sequelae & $\begin{array}{l}\text { Proven new thrombosis, skin necrosis, or } \\
\text { acute systemic reaction after intravenous } \\
\text { unfractionated heparin bolus }\end{array}$ & $\begin{array}{l}\text { Progressive or recurrent thrombosis, } \\
\text { erythematous skin lesions or suspected } \\
\text { thrombosis (not proven) }\end{array}$ & None \\
\hline Other cause(s) of platelet fall & None evident & Possible & Definite \\
\hline
\end{tabular}

\section{Table 2. Baseline characteristics of patients $(N=500)$}

\begin{tabular}{ll}
\hline Parameters & $\boldsymbol{n}(\%)$ \\
\hline Age (years), mean (SD) & $56.8(16.6)$ \\
Male & $330(66.0)$ \\
APACHE II score, mean (SD) & $12.0(7.1)$ \\
Medical history & \\
Hypertension & $222(44.4)$ \\
Diabetes mellitus & $91(18.2)$ \\
Cardiovascular disease & $70(14.0)$ \\
Chronic renal failure & $33(6.6)$ \\
Chronic liver disease & $15(3.0)$ \\
Chronic obstructive pulmonary disease & $31(6.2)$ \\
Neurological disorders & $20(4.0)$ \\
Reason for ICU admission & \\
Respiratory & $90(18.0)$ \\
Sepsis & $301(60.2)$ \\
Fluid overload & $28(5.6)$ \\
Cardiovascular problem & $41(8.2)$ \\
Postoperative & $19(3.8)$ \\
Others & $21(4.2)$ \\
ICU length of stay (days), mean (SD) & $5.8(5.5)$ \\
& $65(13.0)$ \\
&
\end{tabular}

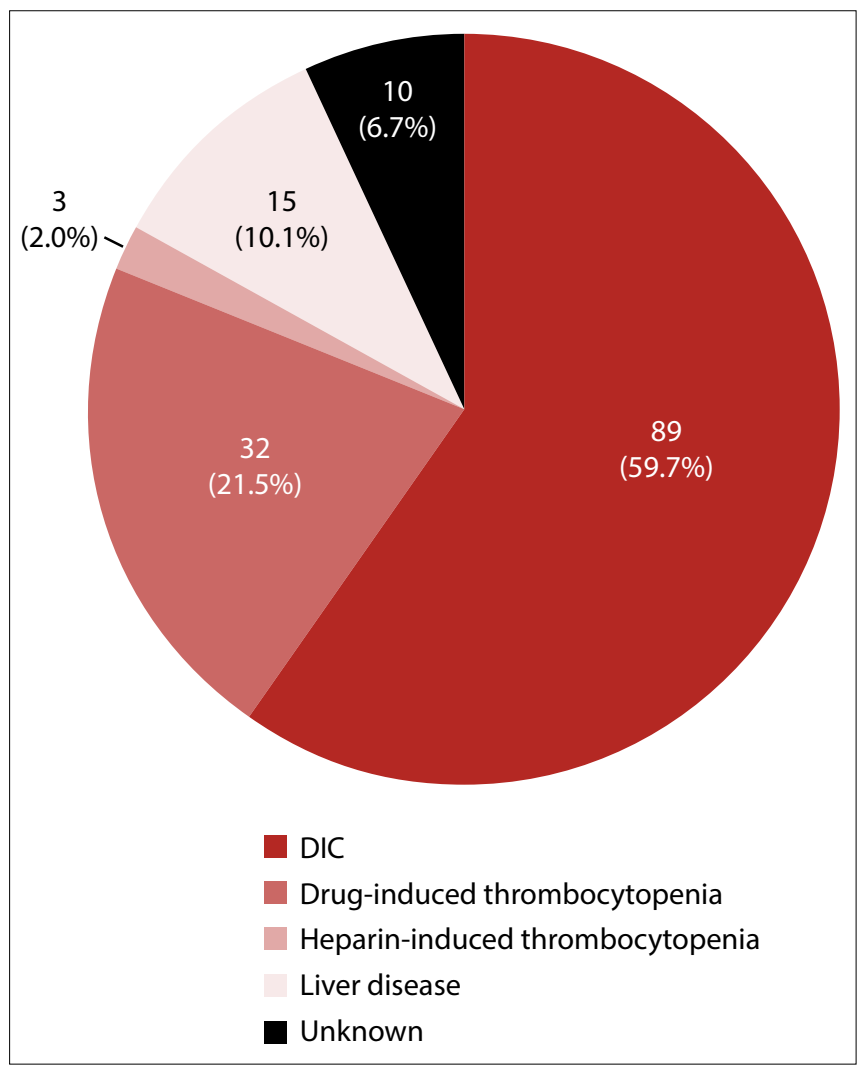

Fig. 1. Causes of new-onset thrombocytopenia. 


\section{Causes of NOT}

A total of 149 patients developed thrombocytopenia during their ICU stay. Thrombocytopenia was diagnosed secondary to sepsis with disseminated intravascular coagulation in 89 patients $(59.7 \%)$ and drug-induced thrombocytopenia was thought to be present in 31 (21.5\%) patients Thrombocytopenia was due to underlying liver disease in $15(10.1 \%)$ patients. HIT was diagnosed, based on $4 \mathrm{~T}$ scoring, in $3(2.0 \%)$ patients. Cause of thrombocytopenia could not be ascertained in $10(6.7 \%)$ patients. During the work-up for NOT, 41 patients refused to give consent for bone marrow examination. Causes of NOT and drug-induced thrombocytopenia are shown in Fig. 1 and Table 3, respectively.

Use of antibiotics, including meropenem, cefuroxime, cefepime, tazobactam, vancomycin, levofloxacin, linezolid, and lowmolecular-weight heparin, correlated with thrombocytopenia, as did underlying coronary artery disease and sepsis $(p<0.05)$.

APACHE II score was significantly higher in patients with NOT compared with those without thrombocytopenia (13 v. 11, $p=0.003$ ). Patients with NOT required significantly more packed red-cell and platelet transfusions (5.4 v. $2.3 \%, p=0.000)$ and had significantly higher ICU mortality ( 15.4 v. $8.7 \%, p=0.003$ ). The majority of transfusions were given prophylactically before procedures in the thrombocytopenia group. Blood products transfused were mainly fresh-frozen plasma and platelets. Patients with NOT required mechanical ventilation and inotropes more frequently (57.7 v. $38.4 \%$, $p=0.000 ; 23.5$ v. $13.5 \%, p=0.008$, respectively).

There was no difference in duration of ICU and hospital stay or bleeding risks between the two groups. Use of invasive devices such as central-line and arterial-line catheters was not different between the groups and haemodialysis requirements also did not vary. Comparison between patients with or without NOT is depicted in Table 4.

\section{Table 3. Causes of drug-induced} thrombocytopenia $(N=32)$

\begin{tabular}{lll}
\hline Cause & $\boldsymbol{n}(\%)$ & $\boldsymbol{p}$-value \\
\hline Carbapenem & $12(8.05)$ & $0.002^{*}$ \\
Cephalosporin & $5(3.35)$ & $0.000^{*}$ \\
Fluoroquinoles & $3(2.01)$ & $0.000^{*}$ \\
Glycopeptides & $6(3.35)$ & $0.002^{*}$ \\
Oxazolidinone & $5(3.35)$ & $0.040^{*}$ \\
$\beta$-lactamase/ & $1(0.67)$ & 0.277 \\
$\beta$-lactamase inhibitors & & \\
${ }^{*} p$-value $<0.05$, statistically significant. &
\end{tabular}

\section{Discussion}

Thrombocytopenia is one of the most common haematological disorders seen in critically ill patients. Prevalence has been found to be around $50 \%$ and incidence varies between $13 \%$ and $44 \%{ }^{[8,9]}$ In our cohort, 41 patients $(8.2 \%)$ had pre-existing thrombocytopenia at the time of ICU admission and 149 patients (32.4\%) developed thrombocytopenia during their ICU stay. Our incidence of $30 \%$ and prevalence of $38 \%$ were consistent with observations made internationally. ${ }^{[6,10,11]}$ This is despite our study definition for thrombocytopenia of two platelet count readings $<150000 / \mu \mathrm{L}$ at least 12 hours apart. This is unlike previous investigations with thrombocytopenia defined as platelet count $<150000 / \mu \mathrm{L}$, or fall in platelet count by at least $50 \%$ from the time of ICU admission.

The majority of studies of thrombocytopenia in ICU are from North America or Europe. Among studies from Asian countries, a prospective study was conducted in Singapore, examining thrombocytopenia incidence in septic critically sick patients only. ${ }^{[12]}$ A study from Korea reported an incidence of $37.1 \%$. Therefore, this is the first prospective study of thrombocytopenia incidence in a general ICU on the Asian subcontinent. As discussed earlier, our figures were comparable with those found internationally.

We discovered underlying coronary artery disease and sepsis on admission as major risk factors for developing thrombocytopenia in critically ill patients. Unlike previous studies, blood transfusions, postoperative status, pulmonary artery catherisation and respiratory failure were not identified as risk factors. ${ }^{[1,13]}$ The association of thrombocytopenia with sepsis can be explained on the basis of an underlying DIC process. Other mechanisms implicated in sepsis-induced thrombocytopenia are immune-mediated platelet destruction and haemophagocytic syndrome. ${ }^{[14,15]}$ An association between coronary artery disease and thrombocytopenia has not been reported before and the underlying pathophysiological mechanism for this observation needs further consideration. It may be related to the use of antiplatelet medication in these patients; however, this needs further investigation.

Systematic and extensive investigations are required to establish the cause of thrombocytopenia in ICU, the aetiology of which is multifactorial and complicatedly linked. The work-up of thrombocytopenia in our study left scope for improvement, as $41(27.5 \%)$ patients refused bone marrow examination. Within these constraints, sepsis with DIC was the most common cause, occurring in $59.7 \%$ of patients. The second-most common cause was drug-induced thrombocytopenia, occurring in $21.4 \%$ of patients. Implicated drugs were mainly antibiotics such as teicoplanin, levofloxacin, cefuroxime, meropenem, vancomycin, linezolid, and cefepime and tazobactam combinations.

Table 4. Comparison between patients with or without NOT

\begin{tabular}{|c|c|c|c|}
\hline Variable & $\begin{array}{l}\text { Patients with NOT } \\
(N=149), n(\%)\end{array}$ & $\begin{array}{l}\text { Patients without NOT } \\
(N=310), n(\%)\end{array}$ & $p$-value \\
\hline Age (years), mean (SD), median & $57.3(16.9), 59$ & $56(16.4), 58$ & 0.454 \\
\hline Male & $99(66.4)$ & $201(64.8)$ & 0.673 \\
\hline ICU stay (days), mean (SD), median & $6.1(5.6), 4$ & $5.3(5.2), 3$ & 0.151 \\
\hline Hospital stay (days), mean (SD), median & $15.0(10.9), 12$ & $17.9(19.4), 11$ & 0.086 \\
\hline APACHE II score, mean (SD), median & $13.0(7.3), 13$ & $10.9(6.8), 11$ & $0.003^{*}$ \\
\hline Haemodialysis & $6(4.0)$ & $20(6.5)$ & 0.530 \\
\hline ICU mortality & $37(15.4)$ & $23(8.7)$ & $0.003^{*}$ \\
\hline Mechanical ventilation & $86(57.7)$ & $119(38.4)$ & $0.000^{*}$ \\
\hline Bleeding risk & $8(5.4)$ & $7(2.3)$ & 0.079 \\
\hline Transfusion requirement & $33(22.1)$ & $29(9.4)$ & $0.000^{*}$ \\
\hline Inotropes & $35(23.5)$ & $42(13.5)$ & $0.008^{*}$ \\
\hline Invasive catheters & $103(69.1)$ & $218(70.3)$ & 0.539 \\
\hline Dialysis & $6(4.0)$ & $20(6.5)$ & 0.239 \\
\hline Coronary artery disease & $15(10.1)$ & $46(14.8)$ & $0.043^{\star}$ \\
\hline Sepsis & $101(67.7)$ & $154(49.6)$ & $0.004^{*}$ \\
\hline Respiratory failure & $39(26.2)$ & $36(11.6)$ & 0.617 \\
\hline Postoperative status & $2(1.3)$ & $14(4.5)$ & 0.132 \\
\hline
\end{tabular}


However, we did not find any associations between thrombocytopenia and anticonvulsants, diuretics or $\mathrm{H} 2$ blockers, as reported in previous studies. ${ }^{[6,11]}$ Establishing drug-induced thrombocytopenia remains difficult, as the diagnostic criteria are based on the recovery of platelet count after the drug is discontinued and there is no gold standard for its diagnosis.

HIT was considered separately from drug-induced thrombocytopenia in our study. Its incidence was found to be $2 \%$, which is in tandem with an internationally reported incidence of $1 \%-5 \% \cdot{ }^{[16]}$ We employed $4 \mathrm{~T}$ scoring for diagnosing HIT because of non-availability of the functional and antigen assays used for detecting HIT antibodies. ${ }^{[17,18]}$ All these tests have moderate to high negative predictive value and a low positive predictive value similar to the $4 \mathrm{~T}$ scoring system. A $4 \mathrm{~T}$ score of $5-8$ on a scale of $0-8$ reflects intermediate to high probability of HIT. The magnitude of HIT in Asian countries has not been systematically studied so far although there have been occasional case reports of HIT from the area.

In our study, thrombocytopenia patients did not have any bleeding episodes. They required more platelet and fresh-frozen plasma transfusions compared with the non-thrombocytopenia group, and these were mostly prophylactic transfusions carried out if any surgical procedures or interventions were planned. Previous studies have reported increased bleeding episodes and more transfusion requirements in critically sick patients with thrombocytopenia. ${ }^{[9,11,19-21]}$ As observed in previous studies, ${ }^{[22]}$ our thrombocytopenia patients had more frequent need for mechanical ventilation and inotropes. We observed a higher ICU mortality among thrombocytopenia patients compared with the non-thrombocytopenia group ( 15.4 v. $8.7 \%, p=0.03)$; this may be because thrombocytopenia patients were much more sick than those who did not develop thrombocytopenia. This correlates with the observation that the APACHE II score at the time of ICU admission was higher in those who eventually developed thrombocytopenia during their ICU stay (13 v. 11, $p=0.003$ ). Thus, thrombocytopenia may reflect severity of critical illness.

Many previous studies have observed poor outcomes with thrombocytopenia. ${ }^{[20,23-27]}$ There have been reports indicating that the rate of platelet decline or recovery from thrombocytopenia may have important prognostic value in diseases such as sepsis and acute respiratory distress syndrome (ARDS). ${ }^{[21,22,24,26]}$

Our study had several limitations. Firstly, this study was conducted at a single tertiary care centre in a northern part of India. Figures may not be truly representative of the entire country. The need for a robust work-up for thrombocytopenia cannot be over-emphasised. This was the second drawback of the study, as evaluation of cause of thrombocytopenia could have been more extensive and systematic. A third limitation was the non-availability of HIT antibody detection tests, which could have influenced the diagnosis.

Major strengths of this study were its prospective nature and adequate sample size.

\section{Conclusions}

Thrombocytopenia occurs frequently in critically ill patients. It should be viewed generally as an indicator of illness severity, and also has prognostic significance because of its association with increased morbidity, hospital resource utilisation and increased mortality. Its potential as a predictor of complications of ARDS and hospital-acquired pneumonia needs to be explored further. However, it remains necessary to evaluate this disorder at the earliest opportunity, as about $25 \%$ of cases are drug-associated and can be rapidly reversed with withdrawal of the offending drug, especially heparin. Rate of decline and delayed count recovery need further evaluation but may prove useful as urgently needed prognostic indicators.

Declaration. The authors declare that an abstract of this study was published in the Indian Journal of Critical Care Medicine as part of a poster presentation at CRITICARE-2015, Bengaluru, India.

References

1. Bonfiglio MF, Traeger SM, Kier KL, Martin BR, Hulisz DT, Verbeck SR. Thrombocytopenia in intensive care patients: A comprehensive analysis of risk factors in 314 patients. Ann Pharmacother 1995;29(9):835-842.

2. Hine LK, Gerstman BB, Wise RP, Tsong Y. Mortality resulting from blood dyscrasias in the United States, 1984. Ann J Med 1990:88(2):151-153.

3. Drews RE. Critical issues in hematology: Anemia, thrombocytopenia, coagulopathy, and blood product transfusions in critically ill patients. Clin Chest Med 2003;24(4):607-622. DOI:10.1016/ s0272-5231(03)00100-x

4. Brooks AP. Thrombocytopenia during treatment with ampicillin. Lancet 1974;2(7882):723. DOI:10.1016/s0140-6736(74)93302-9

5. Kaplan R, Sahn S, Petty T. Incidence and outcome of the respiratory distress syndrome in gram negative sepsis. Arch Intern Med 1979;139(8):867-869.

6. Lim SY, Jeon EJ, Kim H-J, et al. The incidence, causes, and prognostic significance of new-onse thrombocytopenia in intensive care units: A prospective cohort study in a Korean hospital. J Korean Med Sci 2012;27(11):1418-1423. DOI:10.3346/jkms.2012.27.11.1418

7. Warkentin TE. Heparin-induced thrombocytopenia diagnosis and management. Circulation 2004;110(18):e454-e458. DOI:10.1161/01.cir.0000147537.72829.1b

8. Priziola JL, Smythe MA, Dager WE. Drug-induced thrombocytopenia in critically ill patients. Crit Care Med 2010;38(6 Suppl):S145-S154. DOI:10.1097/ccm.0b013e3181de0b88

9. Strauss R, Wehler M, Mehler K, Kreutzer D, Koebnick C, Hahn EG. Thrombocytopenia in patients in the medical intensive care unit: Bleeding prevalence, transfusion requirements an outcome. Crit Care Med 2002;30(8):1765-1771. DOI:10.1097/00003246-200208000-00015

10. Cawley MJ, Wittbrodt ET, Boyce EG, Skaar DJ. Potential risk factors associated with thrombocytopenia in a surgical intensive care unit. Pharmacotherapy 1999;19(1):108-113. thrombocytopenia in a surgical
DOI:10.1592/phco.19.1.108.30518

11. Shalansky SJ, Verma AK, Levine M, Spinelli JJ, Dodek PM. Risk markers for thrombocytopenia in critically ill patients: A prospective analysis. Pharmacotherapy 2002;22(7):803-813. DOI:10.1592/ phco.22.11.803.33634

12. Lee KH, Hui KP, Tan WC. Thrombocytopenia in sepsis: A predictor of mortality in the intensive care unit. Singapore Med J 1993;34(3):245-246.

13. Gupta $\mathrm{LK}$, James B. S74 incidence and analysis of risk factors for thrombocytopenia in critically ill patients. Thorax 2012;67(Suppl 2):A37-A38. DOI:10.1136/thoraxjnl-2012-202678.080

14. Neame PB, Kelton JG, Walker IR, Stewart IO, Nossel HL, Hirsh J. Thrombocytopenia in septicemia: The role of disseminated intravascular coagulation. Blood 1980;56(1):88-92.

15. Stéphan F, Thiolière B, Verdy E, Tulliez M. Role of hemophagocytic histiocytosis in the etiology of thrombocytopenia in patients with sepsis syndrome or septic shock. Clin Infect Dis 1997;25(5):1159-1164. DOI:10.1086/516086

16. Warkentin TE, Cook RJ, Marder VJ, et al. Anti-platelet factor 4/heparin antibodies in orthopedic surgery patients receiving antithrombotic prophylaxis with fondaparinux or enoxaparin. Blood 2005;106(12):3791-3796. DOI:10.1182/blood-2005-05-1938

17. Parker RI. Etiology and significance of thrombocytopenia in critically ill patients. Crit Care Clin 2012;28(3):399-411. DOI:10.1016/j.ccc.2012.04.007

18. Sakr Y. Heparin-induced thrombocytopenia in the ICU: An overview. Crit Care 2011;15(2):211219. DOI:10.1186/cc9993

19. Chakraverty R, Davidson S, Peggs K, Stross P, Garrard C, Littlewood TJ. The incidence and cause of coagulopathies in an intensive care population. Br J Haematol 1996;93(2):460-463 DOI:10.1046/j.1365-2141.1996.5101050.x

20. Vanderschueren S, De Weerdt A, Malbrain M, et al. Thrombocytopenia and prognosis in intensive care. Crit Care Med 2000;28(6):1871-1876. DOI:10.1097/00003246-200006000-00031

21. Moreau D, Timsit J-F, Vesin A, et al. Platelet count decline: An early prognostic marker in critically ill patients with prolonged ICU stays. Chest 2007;131(6):1735-1741. DOI:10.1378/chest.06-2233

22. Venkata C, Kashyap R, Farmer JC, Afessa B. Thrombocytopenia in adult patients with sepsis: Incidence, factors, and its association with clinical outcome. J Intensive Care 2013;1(1):9. DOI:10.1186/2052-0492-1-9

23. Crowther MA, Cook DJ, Meade MO, et al. Thrombocytopenia in medical-surgical critically ill patients: Prevalence, incidence, and risk factors. J Crit Care 2005;20(4):348-353. DOI:10.1016/j. jcrc.2005.09.008

24. Oguzulgen IK, Ozis T, Gursel G. Is the fall in platelet count associated with intensive care unit acquired pneumonia? Swiss Med Wkly 2004;134(29-30):430-434. DOI:2004/29/smw-10670

25. Mokhtari M, Koochak M, Miri M, et al. The incidence of thrombocytopenia and mortality in ICU patients of Imam Hossein hospital. Pejouhesh 2012;36(2):93-99.

26. Wang T, Liu Z, Wang Z, et al. Thrombocytopenia is associated with acute respiratory distress syndrome mortality: An international study. PLoS One 2014;9(4):e94124. DOI:10.1371/journal. pone.0094124

27. Sprung CL, Peduzzi PN, Shatney CH, et al. Impact of encephalopathy on mortality in the sepsis syndrome. The Veterans Administration Systemic Sepsis Cooperative Study Group. Crit Care Med 1990;18(8):801-806 